\title{
Dividend Policy Irrelevancy and the Construct of Earnings
}

James A. Ohlson

W.P. Carey School of Business

Arizona State University
A.J. Ostaszewski

Mathematics Department, London School of Economics,

Houghton Street, London WC2A 2AE

\section{Zhan Gao}

W.P. Carey School of Business

Arizona State University

February 2006

CDAM Research Report LSE-CDAM-2006-23 


\begin{abstract}
In a neo-classical setting of equity-valuation, this paper develops a principle of dividend policy irrelevancy (DPI) to identify and exploit characteristics of earnings. The latter refers to the idea that a value-relevant variable can not reasonably be labeled "earnings" unless it satisfies certain analytical properties with intuitive appeal. The paper proceeds in two parts. The first part, which culminates in Proposition I, provides necessary and sufficient conditions for DPI. The second part concerns how DPI predicates constructs of earnings and their analytical properties. A key result, Proposition II, shows that one can use the analytical properties of earnings to deduce the core approach in practical equity-valuation, namely, measures of growth in expected earnings explain the price to forward-earnings ratio.
\end{abstract}




\section{Introduction}

This paper analyzes dividend policy irrelevancy ("DPI", henceforth) as it relates to constructs, or characteristics, of earnings. DPI, which has long served as a benchmark in financial theory, suggests that the distribution of wealth is of no interest as compared to the (expected) creation of wealth. The claim appeals intuitively since a change in the dividend policy can only influence the sequence of future expected dividends as opposed to their present value. ${ }^{1}$ An investor should not have to forecast next (and later) period's dividends to determine equity value under such circumstances. Instead an investor should forecast future value creation, i.e., she will turn to expected earnings as a practical matter. ${ }^{2}$ This statement, however, may seem much too general unless one identifies what the word "earnings" actually means. Thus the basic question dealt with in the paper arises: Can one exploit DPI to derive intuitively appealing characteristics of "earnings"?

To appreciate the question at hand, one may consider why the absence of DPI militates against linking earnings to value. Value must now reflect the forecasting of next-period dividends in addition to the forecasting of outcomes due to operating activities. In other words, value today depends on the forecasting of the dividend policy, causing obvious problems if one wants to identify a construct of earnings that relates directly to value. Accounting as generally understood does not measure earnings by embedding the dividend policy; a measure of a firm's next period's expected creation of value should depend neither on the (future) dividend policy nor on the next period's expected dividends. Turning these negative observations into their opposite suggests our broad hypothesis that DPI can act as a useful springboard to analyze the way in which 
appropriately identified earnings variables forge links to value. Stated differently, DPI can be potentially be used to construct variables that "look and smell" like earnings; and with the characteristics of earnings in place the analysis can proceed to the earnings-value relation. Assumptions on the characteristics of earnings ought to restrict the ways in which earnings explains value.

With respect to the modeling, the paper relies on the generic linear dynamics found in Ohlson [1990] (see also Christensen and Feltham [2003], Chapter 9): A set of primitives, which completely describe a firm's future states and evolve linearly over time, determine value through the implied sequence of future expected dividends. The analysis then introduces DPI and relates it to earnings via two stages.

The first stage concerns equivalent characterization of DPI. A central result shows that DPI holds if and only if there exists a composite variable, constructed from the primitives, that grows at the discount rate in the absence of dividends. The construction of the composite variable makes no reference to equity value, dividends or the dividend policy. A savings account works similarly: Earnings on a savings account obviously grow at the interest rate when dividends are zero. And this property of a savings account's earnings ensures DPI (the next section develops the point). DPI, therefore, hinges on whether one can construct a variable that satisfies a familiar characteristic of earnings.

Relying on DPI, the second stage of the paper focuses on how to conceptualize earnings. We identify several appealing characteristics that an earnings construct must satisfy, with the following three being the most essential: ${ }^{3}$

- Earnings should be unaffected by contemporaneous dividends as well as by any dividend policy parameters. 
- Earnings will be foregone due to any dividends paid at the start of the period. The discount rate determines the rate of earnings reduction due to dividend distribution. (I.e., $\$ 1$ dividend reduces subsequent earnings by $\$ 0.08$ if the discount rate is $8 \%$ ).

- Earnings grow, on the margin, at a rate determined by the discount factor. (I.e., the partial derivative of period $t+1$ expected earnings relative to period $t$ expected earnings is 1.08 if the discount factor is $8 \%$, keeping all other relevant date $t$ variables constant).

The analysis of earnings starts with an "ideal" construct, namely, "earnings permanent in expectations". It is ideal because its capitalization suffices to determine value; that is, the price to forward-earnings ratio equals the inverse of the discount factor. We then proceed to analyze "accounting earnings", defined by earnings permanent in expectation plus some "error". To develop insights about accounting earnings, the analysis depends on two ideas: (i) accounting earnings will satisfy many of the same characteristics as ideal earnings if one assumes DPI; and (ii) one can assume that the error satisfies certain dynamic properties to forge a link between value and subsequent expected earnings. It is then shown that assumptions on the characteristics of earnings lead to the Ohlson \& Juetter-Nauroth [2005] valuation model. In essence, working from DPI and extractable characteristics of accounting earnings, this analysis captures the core organizing principle of practical equity valuation: Measures of the growth of expected accounting earnings will explain the price to forward-earnings ratio.

An overview of the paper's sections follows:

- Section II motivates the analysis and questions by reviewing a savings account.

- Sections III, IV develop the general model, including the definition of DPI.

- Section V reviews special cases that show up in the literature.

- Section VI provides sufficient and necessary conditions for DPI. 
- Section VII identifies the characteristics of the "ideal" construct of earnings, referred as earnings permanent in expectation.

- Section VIII develops and exploits the characteristics of accounting earnings to deal with the value-accounting earnings relation.

- Section IX analyzes whether the concept of DPI can be extended to free cash flow irrelevancy.

- Section X concludes and summarizes.

\section{Motivating Ideas: The Case of a Savings Account}

This section motivates why an earnings construct ought to depend on DPI. We review the analysis of a savings account to illustrate core issues. ${ }^{4}$ While this setting presumes certainty, subsequent analyses require no such restriction because one can replace known values with expected values. Principles of analysis will remain the same.

Consider the intertemporal earnings dynamic of a savings account:

$$
\hat{x}_{t+1}=R \cdot \hat{x}_{t}-r \cdot d_{t},
$$

where $\hat{x}_{t}$ and $d_{t}$ represent earnings (i.e., interest earned) and dividends (i.e., net withdrawal from the account) respectively. $R=1+r$ defines the discount rate; that is, $R$ shows up in the present value of dividend formula (PVD): $p_{t}=\sum_{\tau=1}^{\infty} R^{-\tau} d_{t+\tau}$. A complete model also requires some dividend policy. To keep matters simple we model a second dynamic equation analogous to [1]:

$$
d_{t+1}=c_{1} \cdot \hat{x}_{t}+c_{2} \cdot d_{t} .
$$

The parameters $c_{1}$ and $c_{2}$ specify the dividend policy. ${ }^{5}$ Combining the two dynamic equations 
with PVD (and the mild regularity condition, ${ }^{6} c_{1}>0$ and $\left|c_{2}\right|<R$ ) implies

$$
p_{t}=\frac{\hat{x}_{t+1}}{r}
$$

regardless of the two dividend policy parameters. Because of this non-trivial property, it makes sense to say that a savings account satisfies DPI.

DPI means that $r$ and $\hat{x}_{t+1}$ (or $\hat{x}_{t}, d_{t}$ ) suffice to determine $p_{t}$. This straightforward way of looking at valuation contrasts with what happens if one actually evaluates $p_{t}$ by feeding an expected dividend sequence $\left\{d_{t+\tau}\right\}_{\tau=1}^{\infty}$ into the PVD formula. The latter evaluation demands $c_{1}$ and $c_{2}$ as input even though they do not influence value. Imposing DPI on valuation does indeed yield a striking consequence: We can forget about $c_{1}$ and $c_{2}$.

The earnings variable $\hat{x}$ satisfies the "familiar" characteristics mentioned in the introduction:

- $\partial \hat{x}_{t} / \partial d_{t}=0$. Contemporaneous dividends have no influence on earnings; in a similar vein, the construct $\hat{x}_{t}$ does not in any meaningful sense depend on $c_{1}$ and $c_{2}$.

- $\partial \hat{x}_{t+1} / \partial d_{t}=-r$. Given the dynamic [1], this marginal impact of dividends on earnings requires (i) to hold.

- $\partial \hat{x}_{t+1} / \partial \hat{x}_{t}=R$. If one puts $d_{t}$ equal to zero, then earnings grow at the rate of $r$.

One can next ask: What characteristics of earnings for a savings account (or the dynamic of such earnings) lead to DPI? To answer this question, consider a more general dynamic in lieu of the one previously introduced:

$$
x_{t+1}=a \cdot x_{t}+b \cdot d_{t}
$$

and where we do not "label" $x_{t}$. We view $x_{t}$ as an instrumental variable necessary to generate the $\left\{d_{t}\right\}_{t+1}$ sequence given some initialization of $\left(x_{t}, d_{t}\right)$. Combing the dynamics [3] and [2] with the present value of dividends formula one derives $p_{t}$ as a linear function of $x_{t+1}$ and $d_{t+1}$; to do so is 
tedious but otherwise straightforward. ${ }^{7}$ Further, if one examines the expression for the two coefficients it becomes apparent that DPI holds if and only if $a=R$. The parameter $b$ does not, perhaps surprisingly, influence DPI given the regularity condition $b \neq 0$.

Taking a closer look at the valuation function helps to understand the nature of DPI. Specifically, the condition $a=R$ implies, and is implied by, $p_{t}$ not depending on $d_{t+1}$. This observation and the ones in previous paragraph underscore that DPI bears on the dynamic $(a=R$ but not $b$ ) as well as the valuation (only $x_{t+1}$ but not $d_{t+1}$ affects $p_{t}$ ) in a precise and complete manner.

Accounting research has always faced the problem of what one can, and cannot, label earnings. In the present context, why not proceed by defining earnings as $x_{t}^{\prime} \equiv r \cdot p_{t-1}$ ? To address the question we simply note that if $a \neq R$, then $x_{t}^{\prime}$ depends on $d_{t}$ and $\left(c_{1}, c_{2}\right)$. Earnings as we know it disallows such dependencies; to refer to $x_{t}^{\prime}$ as earnings would be an abuse of language, at best. One can in any event argue that to define earnings in term of value is awkward. There is a big difference between a definition $x_{t}^{\prime} \equiv r \cdot p_{t-1}$ as opposed to a conclusion $x_{t}=r \cdot p_{t-1}$. And the latter applies if and only if $a=R$. Thus one sees that the first and third characteristics of earnings link up with DPI. The second one, on the other hand, does not. However, it still makes sense to require $b=-r$ since earnings is a flow variable and $-r$ reflects the foregone earnings due to the distribution of wealth at the beginning of the period.

The next section develops a general model where the starting point is a vector of primitive variables instead of a single one $\left(x_{t}\right)$. We generalize the definition of DPI and then proceed to identify equivalent conditions for DPI. 


\section{The General Problem of Dividend Policy Irrelevancy}

Consider the $(n+1) \times(n+1)$ linear dynamic

$$
\mathbf{z}_{t+1}=\mathbf{H} \mathbf{z}_{t}, \quad \text { for } t=1,2, \ldots,
$$

where $\mathbf{z}_{t}=\left[\begin{array}{llll}x_{1 t} & \cdots & x_{n t} & d_{t}\end{array}\right]^{T}$ and $\mathbf{H}$ is a $(n+1) \times(n+1)$ matrix. The vector $\mathbf{z}_{1}$ initiates the dynamic and one infers the sequence of expected dividends from $\left(\mathbf{H}, \mathbf{z}_{1}\right)$ via recursive substitution. It will be convenient to partition $\mathbf{H}$ into three ingredients:

$$
\mathbf{H}=\left[\begin{array}{c:c}
\mathbf{A} & \mathbf{b} \\
\hdashline \mathbf{c}^{T}
\end{array}\right],
$$

where $\mathbf{A}=\left[a_{i j}\right]$ is an $n \times n$ matrix, $\mathbf{b}$ and $\mathbf{c}$ are vectors of dimension $n$ and $n+1$, respectively.

We will refer to $\mathbf{c}$ as the dividend policy vector. To motivate this terminology one can think of $\mathbf{c}$ as a choice-vector. To be sure, though, we do not consider a choice set of feasible $\mathbf{c}$ or argue that the absence of DPI leads to a "practical" problem of optimal dividend policy. The reference to vector $\mathbf{c}$ as a policy vector merely underscores that $\mathbf{c}^{T} \mathbf{z}_{t}$ forecasts $d_{t+1}$.

Let

$$
p_{t}=\sum_{\tau=1}^{\infty} R^{-\tau} d_{t+\tau}
$$

where $R=1+r>1$ specifies the discount factor.

The dynamic [4], along with PVD, raises a basic question which by definition addresses DPI: Fixing $\left(x_{t+1}, d_{t+1}\right)$, under what conditions on $\mathbf{H}$ will $p_{t}$ not depend on the dividend policy vector $\mathbf{c}$ ? That is, though two distinct dividend policy vectors $\mathbf{c}^{\prime}$ and $\mathbf{c}^{\prime \prime}\left(\mathbf{c}^{\prime} \neq \mathbf{c}^{\prime \prime}\right)$ must generally result in $\left\{d_{t}^{\prime}\right\} \neq\left\{d_{t}^{\prime \prime}\right\}$, it may still be true that $p_{t}^{\prime}=p_{t}^{\prime \prime}$ because $\mathbf{H}$ satisfies certain conditions. As we shall see, these yet to be identified conditions are by no means obvious. 


\section{Comments on the Problem}

The dynamic [4] is sufficiently general to handle lagged variables, as is well-known. Expressing [4] as $\mathbf{z}_{t+1}=\mathbf{H}_{1} \mathbf{z}_{t}+\mathbf{H}_{2} \mathbf{z}_{t-1}$ adds no generality. ${ }^{8}$ In particular, the primitive variables (i.e., the $x_{k t}$ ) may be carried over from one date to the next so that, say, $x_{j t}=x_{k t-1}$. Moreover, while $x_{k t} \neq d_{t}$ for all $k=1, \ldots, n$, it could be that $x_{k t}=d_{t-\tau}$, for some $\tau \geq 1$. It is clear that the dependence of $x_{k t}$ on $d_{t-1}, d_{t-2}, \ldots$ can be quite general.

The present value of $\left\{d_{t}\right\}$ will be finite if all roots (or moduli of the roots) of $\mathbf{H}$ are strictly less than $R$. This standard assumption applies throughout when we refer to [4].

Given some $(\mathbf{A}, \mathbf{b})$, the requirement of finite price imposes mild regularity restrictions on vector $\mathbf{c}$. These restrictions, however, will be of no interest to us as we study DPI.

Combing [4] with PVD yields the linear solution

$$
p_{t}=\boldsymbol{\alpha}^{T} \mathbf{z}_{t+1}=\sum_{k=1}^{n} \alpha_{k} x_{k, t+1}+\alpha_{n+1} d_{t+1},
$$

where $\boldsymbol{\alpha}^{T}=\left[\begin{array}{llll}0 & \cdots & 0 & 1\end{array}\right](R \mathbf{I}-\mathbf{H})^{-1}$. DPI means that no elements in $\boldsymbol{\alpha}$ can depend on $\mathbf{c}$, again subject to the assumption of a finite price.

It is suggestive that $\alpha_{n+1}=0$ should be necessary for DPI. After all, if one can infer value without knowing the details of the sequence $d_{t+2}, d_{t+3}, \ldots$, then it would seem contradictory to suggest that one nevertheless needs to know $d_{t+1}$. The question arises to what the extent $\alpha_{n+1}=0$ relates to the DPI condition that $\alpha_{1}, \ldots, \alpha_{\mathrm{n}}$ must be independent of $\mathbf{c}$. We address the necessity and sufficiency of this issue.

One can put $p_{t}=\boldsymbol{\alpha}^{T} \mathbf{Z}_{t+1}$ aside and entertain the somewhat different valuation function $p_{t}=\boldsymbol{\alpha}^{T} \mathbf{H z} z_{t}$. Though the latter expression could have been used to develop the analysis, it would 
not add generality or lead to more useful insights. Hence, we consider the former expression only, in which case the vector $\mathbf{z}_{t+1}$ refers to expected outcomes since value has a date $t$ subscript. ${ }^{9}$ (Had we considered $p_{t}=\boldsymbol{\alpha}^{T} \mathbf{H z}$, then $\mathbf{z}_{t}$ would be viewed as a realized, rather than an expected, outcome.)

When $n$ is relatively small, and/or if $\mathbf{A}$ has many zero entries, direct inversion of the matrix $R \mathbf{I}-\mathbf{H}$ allows for an explicit expression of $\boldsymbol{\alpha}$. Prior research has exploited this aspect of the problem by restricting $\mathbf{H}$ to be $3 \times 3$ with a couple of zeros. We review this research in the next section to illuminate DPI and why it takes on a constructive role in parsimonious models of equity valuation.

\section{Special Cases of the DPI Problem}

Prior research has modeled a variety of accounting-based valuation settings based on the linear dynamic [4] and DPI. These models go beyond a savings account because the dynamics (or Matrix $\mathbf{H}$ ) have a dimension of 3 (or even more). We review three of these settings, though there are many others. In particular, we review a special version of the Free Cash Flows Discounting Model found in Feltham and Ohlson [1995], the standard Market-to-Book Model found in many papers and textbooks (e.g. Penman [2002], pp 145), and the Earnings Growth Model due to Ohlson and Juetter-Nauroth [2005]. An examination of how these models fit into the general Section III problem helps one to better understand various aspects of DPI. It brings out why accounting-based valuation models mesh with DPI.

Consider the following $3 \times 3$ case of [4]: 


$$
\mathbf{H}=\left[\begin{array}{lll}
a_{11} & 1 & b_{1} \\
0 & a_{22} & 0 \\
c_{1} & c_{2} & c_{3}
\end{array}\right],
$$

where $0 \leq a_{22}<R$. The regularity condition $b_{1} c_{1} \neq 0$ ensures that the sequences $\left\{x_{1 t}\right\}$ and $\left\{d_{t}\right\}$ connect with each other. To assume $a_{12}=1$ loses no generality; one can always rescale $x_{2 t}$ so that $a_{12}=1$.

It turns out that for all the three models referred to earlier $a_{11}=R$. This specification builds in DPI; an explicit derivation of $\boldsymbol{\alpha}$ as function of $\mathbf{H}$ shows that $\boldsymbol{\alpha}$ is independent of $\mathbf{c}$, regardless of $b_{1}$. We discuss this point later. Moreover, depending on the value of $b_{1}-$ and $b_{1}$ only - one can identify the three models referred to earlier. With $b_{1}$ specified, one degree of freedom remains -$a_{22}$. Thus, $\boldsymbol{\alpha}$ will be a function of $a_{22}$ and $R$ only, given some explicit numeric value of $b_{1}$.

Model A: $b_{1}=-1$. This specification leads to the simplest version of the "Free Cash Flow Discounting Model" in which free cash flows grow at a constant rate (Feltham and Ohlson [1995]). Read $x_{1 t}$ as net financial assets valued at the end of period $t-1$, denoted by $f a_{t-1}$, and $x_{2 \mathrm{t}}$ as "free cash flows" due to operating (non-financial) activities for period $t$, denoted by $c_{t}$. Valuation reduces to:

$$
p_{t}=f a_{t}+c_{t+1} /\left(R-a_{22}\right) .
$$

Model B: $b_{1}=-R$. This specification leads to the standard Market-to-Book Model (e.g., Penman [2002], pp 145). Read $x_{1 t}$ as cum-dividend book value, denoted by $\overline{b v_{t}}=b v_{t}+d_{t}$, and $x_{2 \mathrm{t}}$ as (expected) residual earnings for period $t+1$, denoted by $x_{t+1}^{a}$. Valuation reduces to:

$$
p_{t}=b v_{t}+x_{t+1}^{a} /\left(R-a_{22}\right) \text {. }
$$

Model C: $b_{1}=-r$. This specification leads to the model that starts from capitalized forward-earnings and complements with a term adjusting for measures of growth in earnings 
(Ohlson and Juetter-Nauroth [2005]). Read $x_{1 t}$ as earnings for period $t$, denoted by $x_{t}$, and $x_{2 t}$ as increments in residual earnings for period $t+1$ vs. $t$, denoted by $\Delta x_{t+1}^{a}$. Valuation reduces to:

$$
p_{t}=x_{t+1} / r+\Delta x_{t+2}^{a} / r\left(R-a_{22}\right)
$$

Though tedious, to identify the solution to the three models does not pose much of a problem given that one solves for $\left(\alpha_{1}, \alpha_{2}, \alpha_{3}\right)$ in case of [6]. This can be done following a routine (though messy) inversion of the matrix $R \mathbf{I}-\mathbf{H}$. [Specifically, $\alpha_{1}=c_{1} / \Delta$, $\alpha_{2}=\left(c_{1}+c_{2}\left(R-a_{11}\right)\right) /\left(\left(R-a_{22}\right) \Delta\right), \alpha_{3}=\left(R-a_{11}\right) / \Delta$, where $\left.\Delta=\left(R-a_{11}\right)\left(R-c_{3}\right)-b_{1} c_{1} \cdot\right]$

Looking at the models through [6] shows why DPI takes on such significance. It cuts by half the number of parameters that affect value. Analyzing equity value thereby becomes more parsimonious than it would be without DPI.

Each model specifies two accounting variables beyond dividends. But the assumptions are sufficiently powerful to ensure that the valuation function ends up only having one degree of freedom, namely, the parameter $a_{22}$. This feature appeals because it captures the idea that growth should make its presence felt in a descriptive valuation model. Depending on interpretations attached to [6], $a_{22}$ specifies the growth of, respectively, free cash flows (Model A), residual earnings (Model B), and the change in residual earnings (Model C).

What assumptions do the three models have in common that leads to their structural simplicity? The answer is clear: DPI, which corresponds to $a_{11}=R$. (The point can be easily verified using the expressions for $\alpha_{1}, \alpha_{2}, \alpha_{3}$ ). One can further show that DPI is equivalent to $\alpha_{3}=0$. This class of models thus demonstrates the three aspects of DPI: (i) the irrelevance of the vector $\mathbf{c}$; (ii) $a_{11}=R$; and (iii) $\alpha_{3}=0$. The next section generalizes these observations.

Finally, note that the saving account is a special case of [6], which eliminates the growth 
parameter in the dynamic. Put $a_{11}=R, x_{2 t} \equiv 0$, and $b_{1}=-r$; one obtains the underlying dynamic $x_{t+1}=R \cdot x_{t}-r \cdot d_{t}$ and the valuation conclusion $p_{t}=x_{t+1} / r$.

\section{The General Settings}

This section develops equivalent characterizations of DPI. As a matter of definition, DPI corresponds to the coefficients of primitives in the valuation, $\boldsymbol{\alpha}$, being independent of the dividend policy vector c. But our prior examples have also shown that the matrix A satisfies certain properties, and further, that the valuation coefficient related to dividends equals zero. The question arises whether we can generalize these aspects of DPI.

To generalize the idea of DPI it helps to see what happens in a specific setting for which one can derive the solution via conjecture. This approach provides more useful insights than what one might think initially.

Consider the matrix

$$
\hat{\mathbf{A}}=\left[\begin{array}{cccc}
a_{11} & 0 & \cdots & 0 \\
a_{21} & a_{22} & \cdots & a_{2 n} \\
\vdots & & & \vdots \\
a_{n 1} & a_{n 2} & \cdots & a_{n n}
\end{array}\right] .
$$

It follows that a conjectured valuation function $p_{t}=K \cdot x_{1 t+1}$ works if, and only if, $a_{11}=R$ in which case again $K=-b_{1}^{-1} .^{10}$ With $a_{11}=R$, the vector $\mathbf{c}$, as well as all the $a_{i j}$, for $i \geq 2$, are irrelevant. The latter parameters influence the sequence of dividends, to be sure, but not their present value (or price).

Mathematically, $a_{11}$ is one of the roots for $\hat{\mathbf{A}}$ (with eigenvector $[1,0, \cdots, 0]$ ). This 
observation suggests that the more general problem revolves around the idea that DPI should be equivalent to $\mathbf{A}$ having one root equal to $R$. Taking this idea one step further, given that $\mathbf{A}$ has a root $R$, one should be able to transform the dynamic [4] into an equivalent representation such that the upper-left partition of the transformed matrix satisfies [7] with $a_{11}=R$. The word "equivalent" means that, subject to the appropriate initialization, the two representations will result in identical sequences of dividends.

The scheme can be applied to diagonalizable $\mathbf{A}$. We use the relation $\mathbf{A}=\mathbf{F}^{-1} \Lambda \mathbf{F}$, where $\mathbf{F}$ is a non-singular $n \times n$ matrix and $\Lambda \equiv\left[\begin{array}{ccc}\lambda_{1} & & \\ & \ddots & \\ & & \\ & & \lambda_{n}\end{array}\right]$ is a diagonal matrix identified by the $n$ roots of $\mathbf{A}$. As indicated, we assume that one of A's roots equals $R$, and without loss of generality put $\lambda_{1}=R$. Now consider the following transformations:

$$
\mathbf{x}_{t} \rightarrow \mathbf{F} \mathbf{x}_{t}, \mathbf{A} \rightarrow \Lambda, \mathbf{b} \rightarrow \mathbf{F b}, \mathbf{c}^{T} \rightarrow \mathbf{c}^{T}\left[\begin{array}{cc}
\mathbf{F}^{-1} & \mathbf{0} \\
\mathbf{0} & 1
\end{array}\right]
$$

The new dynamic is equivalent to the original one. Valuation reduces to

$$
p_{t}=\frac{\hat{x}_{1 t+1}}{-\hat{b}_{1}}=\frac{\mathbf{f}_{1} \mathbf{x}_{t+1}}{-\mathbf{f}_{1} \mathbf{b}}
$$

Here $\mathbf{f}_{1}$ is A's left eigenvector associated with $R$ (the first row of $\mathbf{F}$ ) and it depends only on $\mathbf{A}$. The vector $\mathbf{c}$ is irrelevant, signifying that DPI holds. As to the requirement $\hat{b}_{1} \neq 0$, it means that the $\hat{x}_{1}$-sequence must be influenced by the preceding $d$-sequence. (On a more basic level, the case $\hat{b}_{1}=0$, when $\mathbf{A}$ has a root $R$, violates the condition that the maximum root of $\mathbf{H}$ must be strictly less than $R$; it ensures a finite price. Using a similar argument it can also be shown that the same condition on $\mathbf{H}$ will not be met unless $\hat{c}_{1} \neq 0$ ). 
The structural simplicity of valuation in a setting with DPI becomes apparent. There are four parts to valuation. First, the matrix A must have a root (or eigenvalue) $R$, say $\lambda_{1}=R$. Second, identify the eigenvector associated with $\lambda_{1}, \mathbf{f}_{1}$. Third, transform the $n$-dimensional vector $\mathbf{x}_{t+1}$ to a scalar $\hat{x}_{1 t+1}$ via $\mathbf{f}_{1} \mathbf{x}_{t+1}$. Fourth, introduce the vector $\mathbf{b}$ and complete the valuation function by scaling $\mathbf{f}_{1} \mathbf{x}_{t+1}$ with $-\mathbf{f}_{1} \mathbf{b}$. In a perhaps unexpected way one sees the centrality of the matrix $A$ (it alone determines the root and eigenvector), the subordinate role of $\mathbf{b}$, and the irrelevance of $\mathbf{c}$ in the DPI-based valuation framework.

Not all conceivable A-matrices can be diagonalized. The preceding analysis is therefore suggestive rather than definitive. One can extend the analysis to allow for any $\mathbf{A}$ using the more general but more messy scheme of Jordan canonical form; its implementation would show that the above valuation concepts retain their validity. Here we use a different approach to prove the equivalence of DPI and $\mathbf{A}$ having a root $R$. It relies on $\alpha_{n+1}=0$ to bridge DPI and $\mathbf{A}$ having a root $R$. We follow two steps. First, we prove that DPI is equivalent to $\alpha_{n+1}=0$. Second, $\alpha_{n+1}=0$ is equivalent to $\mathbf{A}$ having a root $R$.

Proposition I. Assume the dynamic [4] and PVD. Then any one of the following three statements implies the remaining two:

(i) DPI holds, i.e., $\boldsymbol{\alpha}$ does not depend on $\mathbf{c}$;

(ii) $\alpha_{n+1}=0$;

(iii) the matrix $\mathbf{A}$ has a root $R$;

Proof: Refer to Appendix I.

The rather technical analysis in the section provides the necessary ingredients to introduce the construct of "earnings". The next section considers earnings in an ideal sense, which in turn will set the stage for analysis of how accounting earnings forges a link to value. 


\section{An "Ideal" Construct of Earnings}

To summarize the previous analysis, the proposition shows that DPI applies if and only if

$$
p_{t}=\hat{x}_{1 t+1} /\left(-\hat{b}_{1}\right) \text {, }
$$

where $\hat{x}_{1 t+1}=\mathbf{f}_{1} \mathbf{x}_{t+1}$ and $\hat{b}_{1}=\mathbf{f}_{1} \mathbf{b}(\neq 0) ; \mathbf{f}_{1}$ is the eigenvector of $\mathbf{A}$ associated with the root $\lambda_{1}$. Moreover, without referring to value or PVD, one obtains the dynamic for $\hat{x}_{1 t}$ :

$$
\hat{x}_{1 t+1}=R \cdot \hat{x}_{1 t}+\hat{b}_{1} \cdot d_{t} .
$$

This expression follows by pre-multiplying $\mathbf{x}_{t+1}=\mathbf{A} \mathbf{x}_{\mathbf{t}}+\mathbf{b} d_{t}$ with $\mathbf{f}_{1}$ and then use $\mathbf{f}_{1} \mathbf{A}=R \mathbf{f}_{1}$ (i.e., $\mathbf{f}_{1}$ is one of A's eigenvectors).

Equation [8] conveys a concrete message: DPI means that there must exist a composite variable - in terms of $\left(\mathbf{A}, \mathbf{x}_{t}\right)$ but not $\left(\mathbf{c}, \mathbf{b}, d_{t}\right)$ - that grows at the rate of $R$ if dividends happen to be zero. This observation suggests that lurking in the background is an idealized construct of earnings.

To develop such earnings construct one naturally exploits on the idea that its capitalization should determine value, i.e., one identifies the variable by defining

$$
\text { epe }_{t+1} \equiv\left(\frac{r}{-\hat{b}_{1}}\right) \cdot \hat{x}_{1 t+1} \text {. }
$$

One reads "epe" as "earnings permanent in expectations". ${ }^{11}$ To justify this terminology, observe that one obtains the valuation conclusion $p_{t}=e p e_{t+1} / r$ from the epe dynamic

$$
\text { epe }_{t+1}=R \cdot \text { epe }_{t}-r \cdot d_{t} .
$$

Hence, $e p e_{t+1}$ and $d_{t+1}$ remain unchanged - or "permanent" -- for all $t$ if and only if the dividend 
policy satisfies $d_{t}=e p e_{t}$. To appreciate that the latter condition indeed is consistent with the intuition of a $100 \%$ payout, notice again that $e p e_{t}$ does not depend on contemporaneous dividends $d_{t}$ or on the dividend policy vector $\mathbf{c}$. DPI is indeed necessary for the epe construct.

Of course, the mathematics for epe is the same as for a savings account. The reason for the new terminology reflects that epe occurs in an underlying setting of uncertainty and, further, that it is a construct derived from the multi-dimensional underpinning $(\mathbf{A}, \mathbf{b})$ and the primitive vector $\mathbf{x}$ (but not $\mathbf{c}, d_{t}$, or $p_{t}$ ). At any rate, the so constructed epe satisfies all the "ideal" characteristics inherent in a savings account.

The construct epe has a unique relationship to dividends depending on whether the dividends are past, contemporaneous, or subsequent. Past dividends influence earnings negatively $\left(\partial e p e_{t} / \partial d_{t-1}=-r\right)$. With respect to contemporaneous dividends, there is no relation, expect through a dividend policy. Future dividends are influenced positively by epe because of epe $e_{t+1}=r \sum_{\tau=1}^{\infty} R^{-\tau} d_{t+\tau}$. But there is no specificity for individual $d_{t+\tau}$ unless one introduces the policy vector c. The three characteristics are noteworthy because they ought to be preserved even if one shifts the analysis from an "ideal" construct of earnings to a more "practical" construct, an idea that the next section develops.

The positive relation between epe $_{t}$ and the subsequent dividend $d_{t+1}$ can be illustrated via the simplest version of [4] and DPI. Consider the bivariate dynamic:

$$
\begin{aligned}
\text { epe }_{t+1} & =R \cdot \text { epe }_{t}-r \cdot d_{t} \\
d_{t+1} & =c_{1} \cdot \text { epe }_{t}+c_{2} \cdot d_{t}
\end{aligned}
$$

The assumption of finite price, i.e., all roots of $\mathbf{H}$ being strictly less than $R$, holds if $c_{1}>0$. As a pleasing and sensible conclusion, for this class of linear dividend policies epe $e_{t}$ must have a positive effect on subsequent dividends $d_{t+1}$. In sharp contrast, $c_{2}$ is totally irrelevant in its sign; the 
parameter merely "complicates" the dividend dynamic.

The careful reader may object that the above construct of epe lacks proper foundation if $\hat{x}_{1 t+1}$ is a stock variable. After all, epe ought to be a flow variable and the re-scaling of a stock variable does not yield a flow variable! But this argument can be dealt with by introducing the (expected) book value and the clean surplus relation (CSR) so that again $p_{t}=e p e_{t+1} / r$ follows. To develop this argument, suppose $\hat{x}_{1 t+1}$ is a stock variable. Then define $b v_{t} \equiv R\left[-\hat{b}_{1}^{-1} \cdot \hat{x}_{t}\right]-d_{t}$ to obtain

$$
p_{t}=R^{-1} \cdot\left(b v_{t+1}+d_{t+1}\right) .
$$

The variable $\left(b v_{t}+d_{t}\right)$, too, does not depend on the date $t$ dividend since $\partial b v_{t} / \partial d_{t}=-1$ :

$$
\frac{\partial\left(b v_{t}+d_{t}\right)}{\partial d_{t}}=-1+1=0 .
$$

Thus "dividends are paid out of book value (but leaves current earnings unaffected)". Using the three relations $p_{t+1}+d_{t+1}=R \cdot p_{t}$ (implied by PVD), $\Delta b v_{t+1}+d_{t+1} \equiv e p e_{t+1}$ (i.e., CSR), and $p_{t}=R^{-1} \cdot\left(b v_{t+1}+d_{t+1}\right)$, one readily derives $p_{t}=e p e_{t+1} / r$. To be sure, the dynamic of $\left(b v_{t}+d_{t}\right)$ can also be transformed back to [9] by invoking CSR. Thus one recovers the "ideal" flow model from the "ideal" stock model.

As a contrast, one cannot recover the "ideal" stock model from the "ideal" flow model. That is, $p_{t}=e p e_{t+1} / r$ does not imply $p_{t}=R^{-1} \cdot\left(b v_{t+1}+d_{t+1}\right)$ or $b v_{t+1}+d_{t+1}=R \cdot b v_{t}$, even if one maintains CSR. To appreciate this conclusion, assume $p_{t}=\left(b v_{t+1}+d_{t+1}+M\right) / R, M$ being an arbitrary constant. It will be still true that $p_{t}=e p e_{t+1} / r$. One can think of $M$ as an "error" in the balance sheets but these errors cancel (in expectation) because the clean surplus relation determines earnings. 


\section{Characteristics of Accounting Earnings}

Any attempt to conceptualize earnings must necessarily rest on an abstract foundation and initially focus on earnings under "ideal", if not "idealized", circumstances. Our development of "earnings permanent expectations" (epe) conforms to this truism, of course. One can then ask whether the prior analysis helps at all to illustrate the relation between earnings and value as a practical matter. This section approaches the problem by analyzing the nature of the difference between accounting earnings and epe, which we refer to as an "error". If the error satisfies DPI, then it turns out that accounting earnings satisfies the same core characteristics as epe. This analysis sets the stage for an analysis of implications of what happens if one adds assumptions on the error. Assuming that the error grows geometrically we can show that Model $\mathrm{C}$ (the Ohlson and Juetter-Nauroth [2005] model) applies. Another slightly different way of proceeding emphasizes the roles of DPI and the earnings characteristics: Given such assumptions it is again the case that Model $\mathrm{C}$ applies.

As indicated in the previous paragraph, it will be useful to start the analysis by defining “accounting earnings" equal epe adjusted for an error:

$$
\text { err }_{t} \equiv a e_{t}-e p e_{t}
$$

Hence,

$$
p_{t}=a e_{t+1} / r-e r r_{t+1} / r
$$

without making any reference (yet) to the characteristics of $a e_{t}$ or $e r t_{t}$, such as DPI or $\partial a e_{t} / \partial d_{t}=0$. Given the dynamic for epe, it follows that

$$
a e_{t+1}=R \cdot a e_{t}+z_{t}-r \cdot d_{t},
$$

where $z_{t} \equiv e r r_{t+1}-R \cdot e r r_{t}$. 
Next, we show that one can reasonably label $a e$ as "accounting earnings" by adding DPI to the above mechanical setup. The term is justified because ae will satisfy "familiar" characteristics that one associates with earnings. Assume that $\operatorname{err}_{t}\left(\right.$ and $\left.z_{t}\right)$ does not depend on the vector $\mathbf{c}$ or on $d_{t}$. The same must now be true for $a e_{t}$. It follows that:

(1) $\partial a e_{t} / \partial d_{t}=0$;

(2) $\partial a e_{t+1} / \partial d_{t}=-r$.

The marginal effect of $a e_{t}$ on $a e_{t+1}$, keeping $e r r_{t}$ and $z_{t}$ fixed, satisfies:

(3) $\partial a e_{t+1} / \partial a e_{t}=R$.

With respect to valuation, i.e., how value relates to the expectation of subsequent accounting earnings and dividends, one has

(4) $\partial p_{t-1} / \partial a e_{t}=1 / r$;

(5) $\partial p_{t-1} / \partial d_{t}=0$.

All of the five characteristics are inherent in epe. In other words, because of DPI, accounting earnings retains the characteristics of an "ideal" notion of earnings without being "ideal" itself.

At first sight, the error-term err, as well as $z$, may seem arbitrary and without any interpretation. Such is not the case, however. Using an identity found in Ohlson [2002] and Ohlson [2005], one can show that $e r r_{t+1}$ relates to the superior growth in expected earnings. Specifically,

$$
p_{t}=\frac{a e_{t+1}}{r}+\frac{1}{r} \sum_{\tau=1}^{\infty} R^{-\tau} z_{t+\tau},
$$

where $z_{t} \equiv \Delta a e_{t+1}-r \cdot\left(a e_{t}-d_{t}\right)$. Thus,

$$
-e r r_{t+1}=\sum_{\tau=1}^{\infty} R^{-\tau} z_{t+\tau}
$$


holds as an identity. The variable $z_{t}$ captures the superior growth of (expected) earnings for period $t+1$, using prior earnings as a reference point. The word "superior" applies because the increment, $\Delta a e_{t+1}$, has been adjusted for the growth attributable to earnings retained, $a e_{t}-d_{t}$, i.e., the term $r \cdot\left(a e_{t}-d_{t}\right)$. Equation [11] thus shows that the present value of all future superior growth equals $-e r r_{t}$. It appeals to intuition that $-e r r_{t}\left(\equiv e p e_{t}-a e_{t}\right)$ relates positively to the present value of superior accounting earnings growth. In contrast to epe, ae cannot pick up all aspects of the future such as the existence of positive NPV investment opportunities. Thus, $-e r r_{t}>0$ and the future positive growth opportunities will be reflected in superior growth in expected accounting earnings.

One extends the above modeling with the simplest possible assumption on the (expected) err-sequence. Suppose that

$$
e r r_{t+1}=\gamma \cdot e r r_{t}, \gamma<R
$$

That is, the errors will grow geometrically in the future due to the general (expected) growth of the firm. It follows that $z_{t}=-(R-\gamma) e r r_{t}$, and thus

$$
z_{t+1}=\gamma \cdot z_{t}
$$

Conversely, the $z_{t}$-dynamic implies the err-dynamic, given regularity conditions. Given these observations and DPI, one has - err $r_{t}=\sum_{\tau=1}^{\infty} R^{-\tau} z_{t+\tau}=z_{t+1} /(R-\gamma)$ so that

$$
p_{t}=\frac{a e_{t+1}}{r}+\frac{z_{t+1}}{r(R-\gamma)}
$$

where equation [10] defines $z_{t+1}$. One interprets $z_{t+1}$ as the near-term, year $t+2$ vs. year $t+1$, dollar value added due to superior growth in expected accounting earnings. The parameter $\gamma$ identifies the long-term growth rate. 
Of course, this analysis is a somewhat different way of looking at Model C in Section IV, with a change in notation $\left(x_{1 t}=a e_{t}, x_{2 t}=z_{t}, a_{22}=\gamma\right)$ to capture two distinct starting points. Section IV's development starts from the matrix $\mathbf{H}$ and then specifies a number of parameters ( $a_{11}=R, a_{21}$ $\left.=0, b_{1}=-r, b_{2}=0\right)$ that leads to the value function. This section, on the other hand, shows that one can introduce three ideas to arrive at the same result: First, DPI; second, an ideal measure of earnings, epe; third, an "error" in epe which grows geometrically. In this way concepts, rather than structural/ empirical assumptions, ground the analysis. To a corresponding degree it is a more appealing approach to the value-earnings relation.

Restricting the errors to satisfy geometric growth could perhaps be viewed as a convenience-assumption. One counters this claim by requiring the ae-variable to satisfy the previous five characteristics. These assumptions force err to grow geometrically.

Proposition II. Assume PVD and the 3-dimentional linear dynamic:

$$
\left[\begin{array}{l}
a e_{t+1} \\
x_{2 t+1} \\
d_{t+1}
\end{array}\right]=\left[\begin{array}{lll}
a_{11} & a_{12} & b_{1} \\
a_{21} & a_{22} & b_{2} \\
c_{1} & c_{2} & c_{3}
\end{array}\right]\left[\begin{array}{l}
a e_{t} \\
x_{2 t} \\
d_{t}
\end{array}\right] .
$$

Further, assume the following five characteristics of earnings: (1) $\partial a e_{t} / \partial d_{t}=0$;

$$
\begin{gathered}
\partial a e_{t+1} / \partial a e_{t}=R ; \text { (3) } \partial a e_{t+1} / \partial d_{t}=-r ;(4) \partial p_{t} / \partial a e_{t+1}=1 / r ;(5) \partial p_{t-1} / \partial d_{t}=0 . \text { Then, } \\
\frac{e r r_{t+1}}{e r r_{t}}=a_{22} \text {, where } e r r_{t} \equiv a e_{t}-e p e_{t} .
\end{gathered}
$$

Proof: Refer to Appendix II.

Corollary. The assumptions imply Model C, i.e., the Ohlson and Juetter-Nauroth model [2005].

Starting with nine degrees of freedom, the five assumptions on earnings thus restrict five parameters and ensure the irrelevance of three parameters. Two restrictions, $a=R$ and $a_{13}=-r$, 
are trite. More complicated are $a_{21}=b_{2}=0$, which depend on assumption (1), (4), (5). Further, because $a_{21}=b_{2}=0$, one can put $a_{12}=1$ without loss of generality (as noted before, it reduces to an issue of scaling). And, of course, the built-in DPI makes $c_{1}, c_{2}, c_{3}$ irrelevant.

The proposition implies that $\operatorname{err}_{t}$ and $x_{2 t}$ (which corresponds to $z_{\mathrm{t}}$ ) grow at the rate of $a_{22}$, the model's single degree of freedom. Referring to the second proposition in Ohlson and Juetter-Nauroth [2005], one can extend this observation about growth. Specifically, given mild restrictions on the asymptotic dividend policy,

$$
\lim _{t \rightarrow \infty} \frac{a e_{t+1}}{a e_{t}}=a_{22}
$$

As a consequence, ae $e_{t}$ epe $e_{t}$ converges to a constant as $t \rightarrow \infty$. That is, in the limit, accounting earnings captures a fixed fraction of "ideal" earnings. This fraction is indeed less than one given a positive long-term growth rate (i.e., $a_{22}>1$ ) and conservative accounting.

We underscore that Proposition II depends only on the assumptions on the characteristics of accounting earnings, putting aside the "framing" assumptions [12] and PVD. Such a conceptual, rather than "structural/empirical", grounding of the proposition makes it more interesting. Moreover, Model C embeds the central organizing principle of practical equity valuation, namely, measures of earnings growth explain the price to forward-earnings ratio. Ohlson and Juetter-Nauroth [2005] expresses Model C as (using our notation)

$$
p_{t}=\frac{a e_{t+1}}{r}\left[\frac{g_{2}-\left(a_{22}-1\right)}{r-\left(a_{22}-1\right)}\right] \text {, }
$$

where $g_{2} \equiv\left(\Delta a e_{t+2}+r \cdot d_{t+1}\right) / a e_{t+1}$ measures the short-term growth in earnings (adjusted for earnings foregone in period $t+2$ due to next-year dividends). Hence, the model has two measures of growth in earnings, $g_{2}$ and $a_{22}$, and these (in addition to $r$ ) explain the price to forward-earnings ratio. We underscore that the derivation here, in contrast to Ohlson and Juetter-Nauroth [2005], 
flows from assumptions on the characteristics of earnings rather than by restricting the matrix $\mathbf{A}$ up front.

Proposition II leads to Model C and the careful reader might have asked how Models A and B fit into the story, if at all. Do these two models also, in fact, depend on the characteristics of accounting earnings as stipulated by the Proposition? The answer is "yes" in the following sense: Model A is a special case of B, and Model B in turn is a special case of Model C. To appreciate that Model B subsumes Model A, one can assume that a firm uses cash accounting. Such accounting implies that $b v=f a$ and abnormal earnings reduce to free cash flows. With respect to the relation between Model B and C, Ohlson [2005] shows that if Model B holds then so does Model C, but the converse is false. Proposition II accordingly provides a completely general approach to equity valuation as long as the matrix $\mathbf{A}$ is of size two.

\section{On the Irrelevance of Free Cash Flows}

Many academics and practitioners of equity valuation emphasize the discounting of expected free cash flows (FCF) instead of dividends. In light of this it makes sense to ask the following question: If we have DPI, why shouldn't we also have FCF irrelevancy in the spirit of DPI? In this context, it is important to note that as a matter of economics DPI predicates the FCF approach to valuation. One irrelevancy may simply lead to the next.

To address the question of FCF irrelevancy, we need to define FCF and align the FCF approach to PVD and DPI. We use the setting of Feltham and Ohlson [1996]. Let

$f a_{t}=$ financial asset, net of financial liabilities;

$f x_{t}=$ net financial incomes. Assume further, in the spirit of MM, that 


$$
f x_{t}=r \cdot f a_{t-1} \text {. }
$$

Free cash flows, $c_{t}$, are then implicitly defined by an accounting that updates $f a_{t}$ :

$$
f a_{t}=f a_{t-1}+f x_{t}+c_{t}-d_{t}
$$

It follows that

$$
f x_{t+1}=R \cdot f x_{t}+r \cdot\left(c_{t}-d_{t}\right),
$$

which, of course, resembles the dynamic of epe $e_{t}$, provided $f x_{t}$ is independent of $c_{t}-d_{t}$. With these assumptions in place -- and these assumptions do indeed suffice --

$$
p_{t}=f x_{t+1} / r+\sum_{\tau=1}^{\infty} R^{-\tau} c_{t+\tau}
$$

So far the analysis has not invoked DPI: one can, at least in principle, assume that $c_{t}$ depends on prior dividends. But such an assumption makes no economic sense. The whole idea motivating the FCF approach is that the forecasting of FCF should be independent of a firm's financial policy (such as borrowing/ lending to make whatever dividend-payment is viewed as desirable). From the Feltham and Ohlson model [1996] it follows that DPI applies as long as the forecasting of expected free cash flows does not depend on current and past dividends.

Now define operating earnings

$$
o x_{t} \equiv e p e_{t}-f x_{t},
$$

where epe is as defined in the previous sections. Of course, $o x$, like epe, defines operating earnings in an "ideal" sense. Unsurprisingly one obtains

$$
p_{t}=f x_{t+1} / r+o x_{t+1} / r
$$

and

$$
o x_{t+1}=R \cdot o x_{t}-r \cdot c_{t} \text {. }
$$

The last equation suggests that the above defined operating earnings implies "FCF irrelevancy" in 
the similar way as DPI. Does FCF irrelevancy apply? The answer is no. This answer underscores that the assumptions have not excluded $\frac{\partial o x_{t}}{\partial c_{t}} \neq 0 !$ This statement holds because, given the definition of $o x_{t}$ and the independence of $c_{t}$ on $f x_{t}, o x_{t}$ depends on $c_{t}$ if and only if $e p e_{t}$ does. And the basic dynamics permits epe $e_{t}$ to depend on $c_{t}$.

One can take the analysis one step further and identify FCF irrelevancy by examining the appropriate submatrix of A. Specifically, if $x_{n t}$ denotes the expected FCF during period $t$, and one grants DPI, then FCF irrelevancy holds if and only if the submatrix

$$
\left[\begin{array}{ccc}
a_{11} & \cdots & a_{1 n-1} \\
\vdots & & \vdots \\
a_{n-11} & \cdots & a_{n-1 n-1}
\end{array}\right]
$$

has a root $R$, provided the regularity condition. The proposition stated in this paper can thus be put to use again.

Model (C) in Section IV provides a direct and simple illustration why DPI applies but FCF irrelevancy does not. Consider the dynamic in Section IV with DPI. That is, put $a_{11}=R, b_{1}=-1$, $x_{1 t}=f a_{t-1}$, and $x_{2 t}=c_{t}$. One obtains

$$
p_{t}-f a_{t}=\sum_{\tau=1}^{\infty} R^{-\tau} c_{t+\tau}=c_{t+1} /\left(R-a_{22}\right)
$$

Now, one can of course define $o x_{t} \equiv r \cdot c_{t} /\left(R-a_{22}\right)$ and combine it with the dynamic $c_{t+1}=a_{22} c_{t} ;$ one obtains

$$
\begin{aligned}
o x_{t+1} & =R \cdot o x_{t}-r \cdot c_{t} \\
p_{t} & =f a_{t}+o x_{t+1} / r
\end{aligned}
$$

which looks much the same as the mathematics for epe. There is a crucial difference, however: while one can deduce that $\partial p e e_{t} / \partial d_{t}=0$, one cannot do the same for $\partial o x_{t} / \partial c_{t}$ because the 
latter derivative equals $r /\left(R-a_{22}\right)$ !

The greater robustness of DPI compared to free cash flows irrelevancy should come as no surprise. From an economic point of view, financial activities, almost by definition, involve zero-NPV activities. Operating activities, on the other hand, are quite different. While on the margin it may allow for a zero-NPV activity, it makes no sense to say that this is the case for all operating activities. Hence, the detailed evolution of free cash flows cannot be disregarded as easily as that of dividends.

\section{Concluding Remarks}

It is no overstatement to say that the concept of a firm's expected earnings (net income or profit) permeates economic analysis. Academic disciplines within the general area of economics, like finance and classical microeconomics, refer to it extensively though they rarely dwell on its precise meaning. And the centrality of forecasted earnings is present in practical investment analysis as well. This apparent demand for concrete roles of earnings suggests that it would be reasonable to address a question that belongs to accounting theory: What are the intrinsic characteristics of earnings that make the concept identifiable and central? In board terms this question motivates the current paper of course.

Questions about the "demand" for an earnings concept leads to the observation that the "supply" is bound to fall short of any ideal measure of earnings. GAAP's earnings, and attempts to reconfigure GAAP's earnings via financial analysis, muddle the pictures because such earnings measures will not build in clear-cut analytical characteristics. As always, theory does not match

perfectly with reality. In our view, however, the complexities of the real world do not negate the 
importance of the questions we have addressed.

This paper deals with the "demand" for earnings by stipulating a condition for DPI. Only if such condition is in place, can one expect a concept of value creation that separates from value distribution. One can construct an "ideal" earnings variable, referred to as earnings permanent in expectation, without any reference to value or the expected dividends. This earnings construct meets perfectly the "demand" for a measure of value because its capitalization suffices to determine value. The result is of interest if for no other reason because it shows how one generalizes the concept of earnings inherent in a savings account.

As to the "supply" of earnings one must recognize that "accounting earnings" capitalization can never provide sufficient information to determine value. There will be "hole" to plug, which presumably means that investors also must forecast the growth in accounting earnings. Such focus on accounting earnings and their growth leads to an analysis of their characteristics. The modeling in this paper handles it by retaining DPI though there is considerable more complexity. An analysis of the accounting earnings so supplied shows that it will satisfy many reasonable characteristics. These are sufficiently powerful to restrict the accounting earnings-value relation. Accounting earnings then provides "useful information" because one can conceptualize the nature of growth in accounting earnings in a parsimonious fashion. In this way accounting earnings provides a "second best" solution to the problem of assessing a firm's intrinsic value. 


\section{Appendix}

\section{The proof of Proposition I.}

The proof proceeds in two parts: The first part shows the equivalency between (i) and (ii); the second part establishes the equivalency between (ii) and (iii). The equivalency between (i) and (iii) then follows immediately.

1. DPI $\Leftrightarrow=>\alpha_{n+1}=0$.

(a) Suppose $\alpha_{n+1}=0$. We need to show that $\left[\alpha_{1}, \ldots, \alpha_{n}\right]$ does not depend on the dividend policy vector $\mathbf{c}$. For any $\mathbf{z}_{t}$, the condition $R p_{t-1}=p_{t}+d_{t}$ implies that

$$
R \boldsymbol{\alpha} \mathbf{z}_{t}=\boldsymbol{\alpha} \mathbf{H z} z_{t}+d_{t}
$$

since we know that the solution to PVD and the dynamic must be linear. Given that $\alpha_{n+1}=0$, it follows that the LHS of [13] equals $R \sum_{k=1}^{n} \alpha_{k} x_{k}$. The RHS equals $\sum_{k=1}^{n} \beta_{k} x_{k}+\beta_{n+1} d_{t}$, where the vector $\boldsymbol{\beta}$ depends at the most on $\mathbf{H}$ and $\boldsymbol{\alpha}$. Moreover, because $\alpha_{n+1}=0$, it is readily verified that $\boldsymbol{\beta}$ does not depend on the vector $\mathbf{c}$. As a direct consequence, the solution to $\alpha_{1}, \ldots, \alpha_{n}$, obtained by making LHS $=$ RHS for all $\mathbf{z}_{t}$, cannot depend on $\mathbf{c}$ either. Thus DPI applies if $\alpha_{n+1}=0$.

(b) Suppose DPI. Given $\mathbf{x}_{t}$ and $d_{t}$, as well as $(\mathbf{A}, \mathbf{b})$, consider two different dividend policies $\mathbf{c}^{\prime} \neq$ $\mathbf{c}^{\prime \prime}$. From [2], one obtains

$$
\begin{aligned}
& p_{t}^{\prime}=\boldsymbol{\alpha}_{n}\left(\mathbf{A} \mathbf{x}_{t}+\mathbf{b} d_{t}\right)+\alpha_{n+1} \mathbf{c}^{\prime}\left[\begin{array}{c}
\mathbf{x}_{t} \\
d_{t}
\end{array}\right], \\
& p_{t}^{\prime \prime}=\boldsymbol{\alpha}_{n}\left(\mathbf{A} \mathbf{x}_{t}+\mathbf{b} d_{t}\right)+\alpha_{n+1} \mathbf{c}^{\prime \prime}\left[\begin{array}{c}
\mathbf{x}_{t} \\
d_{t}
\end{array}\right]
\end{aligned}
$$

where the coefficients $\boldsymbol{\alpha}$ remain the same for $p^{\prime}$ and $p^{\prime \prime}$ due to the definition of DPI. For any given 
$\left(\mathbf{x}_{t}, d_{t}\right)$, DPI also means $p_{t}^{\prime}=p^{\prime \prime}$. It follows $\alpha_{n+1}=0$ by the assumption $\mathbf{c}^{\prime} \neq \mathbf{c}^{\prime \prime}$.

2. $\alpha_{n+1}=0<=>$ A has a root of $R$.

(a) Suppose $\alpha_{n+1}=0$. From [2], one sees that $\alpha_{n+1}$ is the column- $n+1$, row- $n+1$ entry of the matrix $(R \mathbf{I}-\mathbf{H})^{-1}$. Further note the expression for $\alpha_{n+1}$ is

$$
\alpha_{n+1}=\frac{\operatorname{det}\left(R \mathbf{I}_{n}-\mathbf{A}\right)}{\operatorname{det}\left(R \mathbf{I}_{n+1}-\mathbf{H}\right)}
$$

(Refer to S. Lang, A Second Course in Calculus. Addison Wesley, $2^{\text {nd }}$ edit, pp. 517). It immediately follows that $\alpha_{n+1}=0$ implies $\operatorname{det}\left(R \mathbf{I}_{n}-\mathbf{A}\right)=0$. The last statement is equivalent to $\mathbf{A}$ having a root of $R$.

(b) Suppose $\mathbf{A}$ has a root $R$. The conclusion $\alpha_{n+1}=0$ is immediate from [14].

\section{The proof of Proposition II.}

The proof consists of two steps. Step one shows that the assumptions imply the parameterization:

$$
a_{11}=R, a_{12}=1, b_{1}=-r, a_{21}=0, b_{2}=0 .
$$

Step two finishes the proof by showing $e r r_{t+1} /$ err $r_{t}=x_{2 t+1} / x_{2 t}=a_{22}$.

To prove Step one, first note that the dynamic [12] and Assumption (1) imply that the contemporaneous variables $a e_{t}, x_{2 t}$ and $d_{t}$ are independent of each other. Assumptions (2) and (3) immediately implies that $a_{11}=R, b_{1}=-r$. Next, Proposition I implies that Assumption (5) is equivalent to DPI. It follows that $\left[\begin{array}{ll}a_{11} & a_{12} \\ a_{21} & a_{22}\end{array}\right]$ has a root $R$, or equivalently in this case, $\left(R-a_{11}\right)\left(R-a_{22}\right)=a_{12} a_{21}$. Then $a_{21}=0$ because $a_{12} \neq 0$. Further, the PVD solution to the dynamic [12], along with the restriction $a_{21}=0$, implies that 


$$
p_{t}=\frac{1}{r-a_{12} b_{2} /\left(R-a_{22}\right)} a e_{t+1}+k_{2} x_{2 t+1} .
$$

Combining Equation [A. 15] and Assumption (4) yields $b_{2}=0$.

As to the second step, [A. 15] reduces to

$$
p_{t}=\frac{1}{r}\left(a e_{t+1}+r \cdot k_{2} x_{2 t+1}\right) .
$$

DPI implies epe $e_{t+1}=a e_{t+1}+r \cdot k_{2} x_{2 t+1}$. Then, err $t_{t+1}=-r \cdot k_{2} x_{2 t+1}$, by the definition of err. Thus, $e r r_{t+1} /$ err $r_{t}=x_{2 t+1} / x_{2 t}=a_{22}$. 


\section{References}

Beaver, W., 1997. Financial reporting: An accounting revolution, $3^{\text {rd }}$ ed. Englewood Cliffs, NJ: Prentice-Hall.

Black. F., 1980. The magic in earnings: Economic earnings vs. accounting earnings. Financial Analysis Journal: 2-38

Christensen, P., G. Feltham, 2003. Economics of accounting: Volume I - Information in markets. Kluwer Academic Publishers.

Feltham, G., J. A. Ohlson, 1996. Uncertainty resolution and the theory of depreciation measurement. Journal of accounting research 34 (2): 209-234.

Feltham, G., J. A. Ohlson, 1999. Residual earnings valuation with risk and stochastic interest rates. The Accounting Review 74 (2): 165-183.

Hughes, J. S., J. Liu, M. Zhang, 2002. Valuation and accounting for inflation and foreign exchange. Journal of Accounting Research 42 (4): 731-754.

Martin, A. D., V. J. Mizel, 1966. Introduction to linear algebra. McGraw-Hill, Inc.

Ohlson, J. A., 1990. A synthesis of security valuation theory and the role of dividends, cash flows, and earnings. Contemporary Accounting Research 6 (2), 648-676.

Ohlson, J. A., 1991. The theory of value and earnings, and an introduction to the Ball-Brown analysis. Contemporary Accounting Research 8 (1): 1-19.

Ohlson, J. A., 1995. Earnings, book values, and dividends in equity valuation. Contemporary Accounting Research 11 (2): 661-687.

Ohlson, J. A, 1999. Earnings, Book Values, and Dividends in a Stewardship Setting with Moral 
Hazard. Contemporary Accounting Research 12 (3): 525-540

Ohlson, J. A., 2002. Discussion of residual income and value-creation: The missing link. Review of Accounting Studies 7, 247-251.

Ohlson, J. A., 2005. On accounting-based valuation formulae. Review of Accounting Studies 10 (2-3): 323-347.

Ohlson, J. A., Juettner-Nauroth, B., 2005. Expected EPS and EPS growth as determinants of value. Review of Accounting Studies 10 (2-3): 349-365.

Penman, S. 2002. Financial statement analysis and security valuation: $2^{\text {nd }}$ Edition. McGraw Hill.

Penman, S., T. Sougiannis, 1997. The dividend displacement property and the substitution of anticipated earnings for dividends in equity valuation. The Accounting Review 72 (2): 1-21

Penman, S., T. Sougiannis, 1998. A comparison of dividends, cash flow, and earnings approaches to equity valuation. Contemporary Accounting Research 15 (3): 343-383

Penman, S., n. Yehuda, 2005. The pricing of earnings and cash flows and the validation of accrual accounting. Working paper, Columbia University.

Reichelstein, Stefan J, 1999. Asset valuation and performance measurement in a dynamic agency setting. Review of Accounting Studies 4 (3-4): 235-257

Rubinstein, M., 1976. The irrelevancy of dividend policy in an Arrow-Debreu economy. Journal of Finance 31 (4): 1229-1230.

Ryan, S., 1986. Structural models of the price to earnings relation: Measurement errors in accounting earnings. Graduate School of Business, Stanford University.

Yee, K., 2005. Aggregation, dividend irrelevancy, and earnings-value relations. Contemporary Accounting Research 22 (2): 453-480. 
Zhang, X., 2000. Conservative accounting and equity valuation. Journal of Accounting Research 29: 125-149. 


\section{Endnotes}

${ }^{1}$ Ever since pioneering work due to Modiglion and Miller, DPI has long been part of the finance and accounting literature. A few relevant reference may be useful. Rubinstein [1976] provides a standard treatment in a neo-classical model. Christensen and Feltham [2003] in their textbook describes many accounting-based valuation models that embed DPI. They pay considerable attention to the role of financial assets, such as a savings account, which suffices for DPI. This analysis was originally developed in Ohlson [1991] and Feltham and Ohlson [1995]. Hughes, Liu and Zhang [2002] extends this analyses by allowing for inflation. Yee [2005] provides an extensive treatment on why and how DPI restricts admissible valuation functions. Finally, DPI also shows up in the agency theory literature, e.g., Ohlson [1999] and Reichelstein [1999].

${ }^{2}$ Our term "value creation" should be distinguished from "superior value creation". The former refers (broadly) to earnings while the latter refers (broadly) to residual earnings.

${ }^{3}$ Most papers dealing with value state models which satisfy these three characteristics of earnings, e.g., Ohlson [1991], Ohlson [1995], Ohlson and Juettner-Nauroth [2005], Feltham and Ohlson [1995]. These papers do not, however, link these properties to DPI.

${ }^{4}$ Ohlson [1991] relates a savings account to DPI.

${ }^{5}$ Putting $c_{1} \cdot R=-c_{2} \cdot r=K$ for some $K>0$ results in a fixed payout ratio, $d_{t}=K \cdot \hat{x}_{t}$.

${ }^{6}$ DPI combined with the assumption of finite price implies the regularity condition.

${ }^{7}$ The valuation function is $p_{t}=\alpha_{1} x_{t+1}+\alpha_{2} d_{t+1}$, where $\alpha_{1}=R \cdot c_{1} / \Delta$ and $\alpha_{2}=\left(b c_{1}+(R-a) c_{2}\right) / \Delta$, with $\Delta=-b c_{1}+(R-a)\left(R-c_{2}\right)$. 
${ }^{8}$ For example, the dynamic $\begin{aligned} x_{t+1} & =a_{11} x_{t}+a_{12} x_{t-1}+b_{1} d_{t} \\ d_{t+1} & =c_{1} x_{t}+c_{2} x_{t-1}+c_{3} d_{t}\end{aligned}$ can be restated in the form [4] via the

$$
\hat{x}_{1 t+1}=a_{11} \hat{x}_{1 t}+a_{12} \hat{x}_{2 t}+b_{1} d_{t}
$$

redefined state variables $\hat{x}_{1 t}=x_{t}, \hat{x}_{2 t}=x_{t-1}: \hat{x}_{2 t+1}=1 \cdot \hat{x}_{1 t}+0 \cdot \hat{x}_{2 t}+0 \cdot d_{t}$.

$$
d_{t+1}=c_{1} \hat{x}_{1 t}+c_{2} \hat{x}_{2 t}+c_{3} d_{t}
$$

${ }^{9}$ There is no need to express [4] with error terms, i.e.,

$$
\widetilde{\mathbf{z}}_{t+1}=\mathbf{H} \widetilde{\mathbf{z}}_{t}+\widetilde{\boldsymbol{\varepsilon}}_{t+1}
$$

Here we can set $\widetilde{\boldsymbol{\varepsilon}}_{t+1}=0, \widetilde{\mathbf{z}}_{\tau}=\mathbf{z}_{\tau}$, and $\widetilde{\mathbf{z}}_{\tau+1}=\mathbf{z}_{\tau+1} . \quad \mathbf{z}_{t+1}$ reads as expected $\mathbf{z}_{t+1}$ conditional on $\mathbf{z}_{t}$. This way of looking at the state-variables works fine because we link $p_{t}$ to $\mathbf{z}_{t+1}$.

10 To elaborate, note first that PVD corresponds to $p_{t+1}+d_{t+1}=R \cdot p_{t}$. Second, conjecture a solution $p_{t}=K \cdot x_{1 t+1}$. Combining the two expressions yields

$$
K \cdot x_{1 t+2}+d_{t+1}=K\left(R \cdot x_{1 t+1}\right)
$$

But $x_{1 t+2}=R \cdot x_{1 t+1}-r \cdot d_{t+1}$ so that for $K=-b_{1}^{-1}$ one obtains the valuation function. Here we can use the word "the" because by assumption there is a unique solution. Further, note that the scheme collapse when $a_{11} \neq R$.

${ }^{11}$ Ohlson [2005] develops the distinction between earnings permanent in expectation (epe) vs. permanent earnings. The latter implies the former while the converse is false. To see the point, consider

$$
\begin{array}{ll}
\widetilde{x}_{t+1} & =R \cdot x_{t}-r \cdot d_{t}+v_{t}+\widetilde{\varepsilon}_{1, t+1} \\
\widetilde{v}_{t+1} & =
\end{array} .
$$

Here, earnings $\left(=x_{t}\right)$ is permanent if and only if $v_{t} \equiv 0$. However, earnings can be permanent in expectation because 


$$
E_{t-\tau}\left[\widetilde{x}_{t+1}\right]=R \cdot E_{t-\tau}\left[\widetilde{x}_{t}\right]-r \cdot E_{t-\tau}\left[\tilde{d}_{t}\right]
$$

for all $\tau \geq 1$. In our notation, the equation above reduces to

$$
x_{t+1}=R \cdot x_{t}-r \cdot d_{t}
$$

for all future $t$. It is understood that the valuation occurs at a date prior to $t$.

The idea of "permanent earnings" can be traced to Black [1980], Beaver [1997] and others. Ryan [1986] provides the first formalization (from what we know). Ohlson [1995] provides the first comprehensive treatment of the concept, including the formalization of DPI. 